\title{
BMJ Open FLUID study: study protocol for an open-label, single-centre pilot study to investigate the efFect of Lemborexant on sleep management in Japanese sUbjects aged 50 years and older with Insomnia Disorder
}

Ippei Okada (D) , ${ }^{1}$ Kunihiro Iwamoto, ${ }^{1}$ Seiko Miyata, ${ }^{1}$ Akihiro Fujimoto, ${ }^{2}$ Masaki Tanaka, ${ }^{2}$ Manabu Amano, ${ }^{3}$ Nao Matsuyama (D) , ${ }^{3}$ Toshiaki Taoka, ${ }^{4}$ Shinji Naganawa, ${ }^{5}$ Norio Ozaki ${ }^{1}$

To cite: Okada I, Iwamoto $\mathrm{K}$, Miyata S, et al. FLUID study: study protocol for an open-label, single-centre pilot study to investigate the effect of Lemborexant on sleep management in Japanese sUbjects aged 50 years and older with Insomnia Disorder. BMJ Open 2021;11:e054885. doi:10.1136/ bmjopen-2021-054885

- Prepublication history for this paper is available online. To view these files, please visit the journal online (http://dx.doi. org/10.1136/bmjopen-2021054885).

$\mathrm{IO}$ and $\mathrm{KI}$ contributed equally.

$\mathrm{IO}$ and $\mathrm{KI}$ are joint first authors.

Received 27 June 2021

Accepted 25 0ctober 2021

Check for updates

(C) Author(s) (or their employer(s)) 2021. Re-use permitted under CC BY-NC. No commercial re-use. See rights and permissions. Published by BMJ.

For numbered affiliations see end of article.

Correspondence to

Dr Kunihiro Iwamoto;

iwamoto@med.nagoya-u.ac.jp

\section{ABSTRACT}

Introduction Bidirectional associations have been reported between sleep disturbance and both cognitive impairment, including Alzheimer's disease and amyloid beta-peptide $(A \beta)$ accumulation. These relationships can be explained by the glymphatic system, which acts as a garbage drainage system in the brain. As interstitial fluid dynamics are suggested to increase during sleep, clearance of $A \beta$ can be influenced by sleep disturbance or deprivation. We hypothesised that using lemborexant, an orexin receptor antagonist, to improve sleep quality would also improve the function of the glymphatic system. We plan to examine the effect of lemborexant on sleep quality and the glymphatic system among patients with insomnia disorder.

Methods and analysis This pilot study is designed as an open-label, single-arm, single-centre trial. Thirty patients aged 50 years and over with insomnia will be recruited. The participants will take lemborexant $(5 \mathrm{mg})$ at bedtime for 12 weeks and undergo a home-based sleep study at baseline and weeks 4 and 12, as well as MRI examinations to evaluate the glymphatic system at baseline and week 12. The primary outcome will be changes in objective sleep parameters as evaluated using a sleep monitoring system. The secondary outcomes will be changes in subjective sleep parameters. The relationships between changes in sleep parameters and the glymphatic system will be evaluated using diffusion tensor image analysis along the perivascular space, which is called the ALPSindex. Sleep parameters and the ALPS-index will be analysed using a paired t-test or Pearson's correlation coefficient.

Ethics and dissemination The study protocol was approved by Nagoya University Certified Review Board. The findings from this research will be published in peerreviewed journals and be presented at local, national and international conferences.

Trial registration number $\mathrm{jRCTs041210024.}$
Strengths and limitations of this study

- This is the first prospective trial for elucidating the relationship between sleep and the glymphatic system in humans.

- New insights regarding the relationship between insomnia medication and the glymphatic system will be obtained.

- The glymphatic system will be evaluated using a new noninvasive method-diffusion tensor image analysis along the perivascular space-in patients with insomnia disorder.

- Sleep parameters will be longitudinally evaluated for 12 weeks by an ambulatory sleep electroencephalogram monitor correlated with polysomnography and an electronic sleep diary.

- The results from this study may be considered preliminary because of the small sample size and the non-randomised, placebo-controlled design.

\section{INTRODUCTION}

Chronic insomnia is a common disorder and its prevalence has been estimated to be approximately $10 \%-15 \%$ in the general population. ${ }^{1}$ Furthermore, population-based studies conducted in various countries have found that approximately $30 \%$ of adults have at least one symptom of insomnia, ${ }^{2}$ and that the prevalence is higher among women and older populations. ${ }^{3}$ Insomnia is known to increase the risks for various physical disorders such as hypertension, diabetes, obesity and dyslipidaemia. ${ }^{2}$ Furthermore, insomnia increases the risk of developing depression, ${ }^{4}$ industrial accidents, ${ }^{5}$ traffic accidents, ${ }^{67}$ decreased brain activity ${ }^{8-10}$ and cognitive impairments. 811 
Furthermore, it is becoming clear that the link between degenerative dementia conditions and sleep disruption is bidirectional. Patients with cognitive impairment or dementia have a high prevalence of sleep disturbance. ${ }^{12}$ On the other hand, sleep disturbance contributes to cognitive decline and might also heighten the risk of Alzheimer's disease (AD) by increasing the amyloid betapeptide (A $\beta$ ) burden. ${ }^{13} 14$ A previous positron emission tomography study revealed that older persons with shorter subjective sleep duration had more $A \beta .^{15}$ In addition, increased $A \beta$ is likely to result in sleep abnormalities. ${ }^{16}$

Recently, a mechanism described as the glymphatic system, ${ }^{17}$ which excretes waste products in the cerebral parenchyma into the paravenous efflux pathway, has been proposed. In the glymphatic system, aquaporin-4 water channels densely expressed along astrocytic end-feet mediate the transportation of cerebrospinal fluid (CSF) from the periarterial space to the interstitium, where CSF encounters interstitial fluid (ISF) ${ }^{18}$ Then, the mixed fluid moves to perivascular or perineuronal space. This process results in the clearance of the extracellular 'garbage' from the cerebral parenchyma. It has been suggested that impaired function of the glymphatic system is associated with the onset of neurodegenerative disorders. ${ }^{19}$ In an animal model, clearance of $\mathrm{A} \beta$ during sleep was shown to be faster than that during the waking state, ${ }^{20}$ and sleep deprivation for one night caused the accumulation of $\mathrm{A} \beta,{ }^{21}$ particularly with slow-wave sleep interruption. ${ }^{182}$ This is because the extracellular space expands during sleep, and this expansion may increase the volume of ISF passing through the brain parenchyma, thereby clearing by-products more effectively. ${ }^{23}$ Sleep deprivation or fragmentation can cause increased neuronal activity, leading to elevated $\mathrm{A} \beta$ production and aggregation. Wakefulness also increases sympathetic output and suppresses glymphatic system function, which can result in decreased clearance of pathogenic proteins such as $\mathrm{A} \beta$, tau, or synuclein.

The glymphatic system can be visualised by several new technologies and methods. A new method called diffusion tensor image analysis along the perivascular space (DTI-ALPS) has been developed to evaluate the effectiveness of the glymphatic system. ${ }^{24}$ Recently, a study that used dynamic contrast-enhanced MRI showed that patients with idiopathic Parkinson's disease had an impaired meningeal lymphatic system, which constitutes a drainage system in the brain along with the glymphatic system. ${ }^{25} \mathrm{~A}$ new term 'central nervous system (CNS) interstitial fluidopathy', which includes AD, Parkinson's disease, traumatic brain injury, stroke, small vessel diseases, glaucoma, Ménière's disease and idiopathic normal pressure hydrocephalus, has been proposed. ${ }^{26} \mathrm{~A}$ new method such as DTI-ALPS could provide important new information regarding the relationship among sleep, the glymphatic system and related diseases like CNS interstitial fluidopathy.

Three main types of hypnotics are used in pharmacological treatment for insomnia: benzodiazepine receptor agonists (BzRAs), melatonin receptor agonists and orexin receptor antagonists. BzRAs, which have been used frequently for decades, are usually classified by their elimination half-life. BzRAs reduce sleep latency and wake after sleep onset (WASO) and increase total sleep time (TST) ${ }^{1}$ and act through gamma-aminobutyric acid receptors, which are distributed throughout a broad area of the brain. Thus, BzRAs have anxiolytic, anticonvulsant and muscular relaxant effects; however, they could also induce dependency and increase the risk of falls and cognitive impairment. ${ }^{27-29}$ Recently, an epidemiological meta-analysis showed that a history of BzRA use was significantly associated with the incidence of dementia, and this association was maintained after adjusting for protopathic bias, depression, anxiety and insomnia. ${ }^{30}$ Although the relationship between BzRA use and dementia is not always consistent, ${ }^{31} 32$ a physician should avoid prescribing BzRAs as much as possible. On the other hand, melatonin receptor and orexin receptor antagonists are associated with fewer adverse effects and have become recognised as safer hypnotics compared with BzRAs. The newest approved orexin receptor antagonist, lemborexant, has been shown to improve objective/subjective sleep parameters such as sleep-onset latency (SOL) as measured by polysomnography (PSG) and by patient-reported sleep diaries, WASO and sleep efficiency (SE) ${ }^{33}$ but not to cause rebound insomnia or withdrawal. ${ }^{34}$ In addition, the network metaanalysis revealed the efficacy and safety of lemborexant for patients with insomnia. ${ }^{35}$ However, whether the amelioration of insomnia leads to improvements in the glymphatic system remains unknown.

Therefore, we planned a prospective study, which we named the FLUID study, to clarify the relationship between sleep and the glymphatic system in patients with insomnia. First, we will confirm the effectiveness of lemborexant for improving objective sleep parameters in patients with insomnia using Zmachine (General Sleep, Cleveland, Ohio, USA), a new home-based sleep monitoring system, and validated using PSG. Zmachine can assess patients' natural sleep compared with PSG, because it uses only one channel, and thus imposes a less onerous burden during the night, particularly on patients with insomnia. Second, we will examine the relationship between improvements in sleep and changes in the glymphatic system as evaluated by DTI-ALPS. ${ }^{24}$

\section{METHODS AND ANALYSIS}

\section{Patient and public involvement}

The development of the research questions, outcome measures, study design, recruitment and conduct of the study is not based on patient or public involvement.

\section{Study design}

This pilot study is planned as an open-label, single-arm, single-centre trial. The study protocol was devised under the consideration of the Standard Protocol Items: Recommendation for Interventional Trials guidelines. ${ }^{36}$ This trial will be conducted at Nagoya University Hospital in Japan from 1 June 2021 to 31 October 2023. Patients will take lemborexant at bedtime for 12 weeks and undergo a 
home-based sleep study at baseline and weeks 4 and 12. In addition, they will undergo an MRI examination to evaluate the brain clearance system at baseline and week 12 .

\section{Participants}

The inclusion criteria are: (1) able to provide written informed consent before the study begins, (2) age 50 years and older, (3) insomnia disorder based on the Diagnostic and Statistical Manual of Mental Disorders, Fifth Edition (DSM-5) and (4) score $\geq 24$ on the Japanese version of the Mini-Mental State Examination (MMSE-J). The exclusion criteria are: (1) a history of hypersensitivity to lemborexant, (2) severe hepatic dysfunction (Child-Pugh classification C), (3) a history of lemborexant use, (4) a history of hypnotics use within 1 week before providing consent, (5) a psychiatric illness interfering with scheduled examinations during the study protocol, (6) sleep disorders other than insomnia disorder, such as obstructive sleep apnoea requiring the use of continuous positive airway pressure, periodic limb movement disorders, or restless legs syndrome (RLS), (7) a history of narcolepsy, (8) dementia, (9) specific conditions such as repeated prolonged QT/QTc $\geq 450 \mathrm{~ms}$ on ECG, respiratory disorders, severe hepatic/renal/gastrointestinal disorders, neurological or psychiatric disorders, cancer within the past 5 years or chronic pain, (10) breastfeeding or pregnant female, (11) refusal to use contraception throughout the study period, (12) contradictions for MRI such as a pacemaker, implantable cardioverter-defibrillator, artificial joint or claustrophobia, (13) contradictions for gadolinium (Gd) contrast agents such as a history of allergic reactions and asthma, and (14) judged unsuitable for participation by a physician. The discontinuance criteria are: (1) proposal to discontinue or withdraw consent to participate in the study, (2) emergence of a serious safety problem, (3) a critical event that requires an operative intervention, (4) pregnancy, (5) unable to visit the hospital, (6) judged unsuitable for participation by a principal investigator or coinvestigator, (7) judged ineligible for participation after the beginning of treatment and (8) the discontinuation or interruption of part or all of the study protocol.

\section{Study drug}

Participants will be dispensed lemborexant ( $5 \mathrm{mg}$ ) to be orally administered at bedtime for up to 12 weeks. If a participant feels that the dosage is not effective, it will be increased to $10 \mathrm{mg}$ after consulting with a study physician based on the participant's subjective complaints. Although scheduled visits are set at weeks 4 and 12, additional visits are acceptable according to participant's request. Randomisation and blinding are not required for this study.

\section{Restricted medications}

The following medications will be prohibited during the study: antipsychotics, moderate to strong inhibitors of cytochrome P450 3A4 (CYP3A4), inducers of CYP3A4, anticholinergic drugs, sedative anticonvulsants, sedative anxiolytics, hypnotics such as melatonin and over-thecounter drugs, sedative herbs, sedative muscle relaxants, antihistamines except for non-sedative antihistamines, and other drugs that could induce or worsen sleep disorders as judged by a principal investigator or coinvestigator. The dosages of any antidepressants and mood stabilisers prescribed before the screening period can remain unchanged until the last observation. Other drugs except prohibited drugs will be permitted for temporal use only. Psychotherapies such as cognitive behavioural therapy are not prohibited if the therapy is ongoing.

\section{Test schedule}

Table 1 shows the test schedule for this study. In the screening period, background characteristics, vital signs, results from medical examinations, blood and urine tests and ECG recordings will be assessed.

Sleep-related symptoms, cognitive functions and psychiatric illness will be checked using the rapid eye movement sleep behaviour disorder screening questionnaire, RLS symptom screening tools, and Structured Clinical Interview for DSM-5 screening module. Furthermore, to exclude sleep disorders except for insomnia disorder, participants will undergo a home-based sleep test using Zmachine, portable electromyography (logger; GC, Tokyo, Japan), and pulse oximetry (PULSOX-Me300; Teijin Pharma, Tokyo, Japan). In addition, the participants will complete an electronic sleep diary from the screening period through week 12 .

In the test period, all participants will visit our hospital on three pairs of two successive days at baseline and weeks 4 and 12 after treatment. All participants will undergo the homebased sleep test using Zmachine for a night surrounding the hospital visits. Questionnaires such as the Pittsburgh Sleep Quality Index (PSQI), Epworth Sleepiness Scale (ESS), Beck Depression Inventory (BDI) and Temperament and Character Inventory Harm Avoidance (TCI-HA) will also be conducted. Scores on the Continuous Performance Test, Identical Pairs version (CPT-IP), Wisconsin Card Sorting Test (WCST), Trail Making Test (TMT) and MMSE-J will be analysed, along with eye movement estimation. In addition, blood and urine tests and intestinal flora will be analysed. As optional procedures, participants who provide consent will undergo a Gd-enhanced MRI examination on the evening and following morning at baseline and week 12 after beginning treatment.

\section{Sleep evaluation}

Objective sleep parameters at home will be evaluated using Zmachine, which is a single-channel EEG that monitors sleep and provides algorithm-based sleep staging. Validation studies of Zmachine have been conducted in healthy subjects, patients with insomnia and psychiatric patients. ${ }^{37-39}$ The detailed methods of Zmachine have been reported. ${ }^{37}$ Participants can easily attach Zmachine at night by themselves. The following four parameters will be evaluated: latency to persistent sleep (LPS; elapsed minutes until the beginning of the period in which 10 of $12 \mathrm{~min}$ are scored as sleep) as objective SOL, WASO (total awake minutes following LPS), TST (accumulation of all epochs determined to represent sleep) and SE 
Table 1 Test schedule

\begin{tabular}{|c|c|c|c|c|c|c|c|c|c|}
\hline \multirow{2}{*}{$\begin{array}{l}\text { Schedule } \\
\text { Day }\end{array}$} & \multirow{2}{*}{$\begin{array}{l}\begin{array}{l}\text { Screening } \\
\text { period }\end{array} \\
\begin{array}{l}\text { Within } 28 \\
\text { days }\end{array} \\
\end{array}$} & \multirow{2}{*}{\multicolumn{2}{|c|}{$\begin{array}{l}\text { Baseline } \\
\text { Week } 0\end{array}$}} & \multicolumn{4}{|c|}{ Lemborexant administration period } & \multirow{2}{*}{$\begin{array}{l}\begin{array}{l}\text { Follow-up } \\
\text { period }\end{array} \\
\text { Week } 16 \\
\text { Day } 113 \\
\end{array}$} & \multirow[b]{2}{*}{ Discontinuation } \\
\hline & & & & \multicolumn{2}{|l|}{ Week 4} & \multicolumn{2}{|l|}{ Week 12} & & \\
\hline Acceptable days & & - & - & \pm 7 & \pm 7 & \pm 14 & \pm 14 & \pm 14 & \pm 14 \\
\hline $\begin{array}{l}\text { Participant } \\
\text { background }\end{array}$ & $\circ$ & & & & & & & & \\
\hline Electromyograph & $\circ$ Night & & & & & & & & \\
\hline $\begin{array}{l}\text { Lemborexant } \\
\text { administration }\end{array}$ & & & & 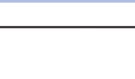 & & 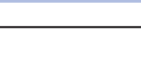 & 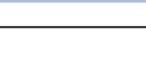 & 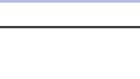 & $\longrightarrow$ \\
\hline Zmachine & $\circ$ Night & ○ Night & & $\circ$ Night & & ○ Night & & & \\
\hline Sleep diary & 4 & 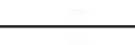 & & & & & & $\rightarrow$ & \\
\hline Cognitive function & & & $\circ$ Morning & & $\circ$ Morning & & $\circ$ Morning & & \\
\hline $\begin{array}{l}\text { Mini-Mental State } \\
\text { Examination }\end{array}$ & $\circ$ & & & & & & $\circ$ Morning & & \\
\hline Questionnaire & & $\circ$ Evening & & $\circ$ Evening & & $\circ$ Evening & & & \\
\hline $\begin{array}{l}\text { Eye movement } \\
\text { analysis }\end{array}$ & & & $\circ$ Morning & & $\circ$ Morning & & $\circ$ Morning & & \\
\hline Intestinal flora test & & & (optional) & & (optional) & & (optional) & & \\
\hline Vital signs & $\circ$ & & & & & & & & $\circ$ \\
\hline Clinical examination & $\circ$ & & & $\circ$ & & $\circ$ & & & $\circ$ \\
\hline ECG & $\circ$ & & & & & & & & $\circ$ \\
\hline
\end{tabular}

(proportion of time spent asleep per time in bed, calculated as TST/total recording time) scores. Subjective sleep parameters will be derived from electronic sleep diaries, and subjective SOL (sSOL), subjective WASO (sWASO), subjective TST (sTST) and subjective SE (sSE) scores will be evaluated. Electronic sleep diaries will encourage the participants to provide complete daily data because an automatic alert message will send them a reminder within a short time after awakening in the morning.

\section{Brain clearance system}

The brain clearance system, known as the glymphatic system, can be evaluated using DTI-ALPS. ${ }^{24}$ To evaluate the activity of the glymphatic system in each participant, the ALPS-index will be calculated. The method for calculating the ALPS-index has been described elsewhere. ${ }^{24}$ Image acquisition will be completed using a 3T clinical scanner (Vantage Centurian; Canon Medical Systems, Tochigi, Japan). DTIs will be acquired simultaneously in addition to conventional morphology images. As another method to evaluate the activity of the glymphatic system, dynamic contrast-enhanced MRI using Gd contrast agent will be performed, ${ }^{40}$ where $\mathrm{K}_{\text {trans }}$ is the volume transfer constant of Gd contrast agent from blood plasma to the extravascular extracellular space, ${ }^{41}$ reflecting both blood plasma flow and permeability; the precise method has been described elsewhere. ${ }^{42} 43$

\section{Questionnaires}

The PSQI will be used to assess the participants' sleep quality and disturbance during the past month. The PSQI is composed of 19 questions covering seven components, including subjective sleep quality, sleep latency and use of sleeping medication. ${ }^{44}$ A participant with a PSQI score $\geq 5$ is regarded as having sleep disturbance. The ESS will be used to assess subjective daytime sleepiness. A participant with an ESS score $\geq 11$ is regarded as having daytime sleepiness. ${ }^{45}$ The BDI-II is practical for research and will therefore be used to assess the severity of depression. ${ }^{46}$ The TCI-HA, which consists of the 20 items, is a HA subscale from the TCI125. ${ }^{47}$ The scales pertain to the following four components: anticipated worry, fear of uncertainty, shyness and fatigability. Participants with high scores tend to be shy, easily fatigued or apprehensive.

\section{Cognitive function}

The CPT-IP will be used to measure sustained attention. ${ }^{48}$ A series of four-digit stimuli are presented for $50 \mathrm{~ms}$, with an interstimulus interval of $950 \mathrm{~ms}$. Each completed task consists of 150 trials, of which, 30 are target trials requiring a response. In this test, performance is measured by the hit 
rate, false rate and signal detection index d-prime, which is a measure of discriminability computed from 'hits' and 'false alarms'. A modified computerised version of the WCST will be administered to measure executive function, and the test lasts until such time as 48 cards are sorted. ${ }^{49}{ }^{50}$ Performance will be measured by the following indices: category achievement, perseverative errors of Nelson and difficulty of maintaining set. TMT parts A and B will be used to measure psychomotor speed, attention, visual scanning ability and executive functioning. ${ }^{51}$ In part A, participants are instructed to connect an ascending series of circles containing numbers as quickly as possible. In part B, participants are instructed to alternate between numbers and letters. The performance measure in these tests is the time required to complete each condition. The MMSE-J, which only focuses on cognitive aspects spoiling the mood, thinking and mental experiences, will be used to assess general cognitive function. ${ }^{52}$

\section{Eye movement analysis}

Measures of eye movement have been applied to evaluate higher brain functions such as cognition, social behaviour and higher-level decision making. Recently, a growing body of research has described eye movements in relation to psychiatric disorders. ${ }^{53} 54$ Eye movement analysis in insomnia has focused on the aspects of mood regulation and emotional information processing. ${ }^{55} \mathrm{We}$ will investigate how sleep improvement by lemborexant changes eye movements.

Methods for eye movement recordings and the processing and analysing of eye movement data have been described elsewhere. ${ }^{56}$ The participants will face a 19-inch liquid crystal display monitor placed $70 \mathrm{~cm}$ in front of their eyes. Visual stimuli will be presented using MATLAB (MathWorks, Natick, Massachusetts, USA) via the Psychophysics Toolbox extension. Eye movements and pupil areas of the left eye will be measured at 1 $\mathrm{kHz}$ using the EyeLink 1000 Plus system (SR Research, Ontario, Canada). The data will be analysed using computer programmes based on MATLAB.

On the basis of a previous study, ${ }^{56}$ we will administer three eye movement examinations to extract three eye movement measures. The free viewing test will be performed using 20 original images involving pictures, geometric patterns and noises. The participants will be instructed to view each presented image freely for $8 \mathrm{~s}$. In the smooth pursuit test, participants are required to track a target moving along a Lissajous trajectory for $20 \mathrm{~s}$. The trial will be repeated twice. In the fixation stability test, participants are required to maintain their gaze on a fixation target presented at the centre of the monitor for $5 \mathrm{~s}$ in the presence of a distractor stimulus that suddenly appears to the left or right of the fixation target. Each condition is repeated twice, and a total of four trials are conducted in random order. The participants will take this test in the morning at baseline and weeks 4 and 12 .

\section{Intestinal flora test}

There is considerable evidence showing that the gut microbiome not only affects the digestive, metabolic and immune functions of the host, but also regulates host sleep and mental states through the microbiome-gutbrain axis. ${ }^{57}$ Gut microbiota are known to differ by age, sex and race ${ }^{58}$; therefore, we will investigate the relationship between insomnia symptoms and the structure of the gut microbiota in the Japanese population.

Participants who consent to this optional substudy will collect faecal samples at home. After extracting total DNA from the samples, long-read metagenomics and $16 \mathrm{~S}$ ribosomal RNA gene PCR will be used to evaluate the gut microbiota. ${ }^{6061}$ Faecal samples will be collected in the morning at baseline and weeks 4 and 12 .

\section{Primary outcome}

The primary outcome is the amount of change in objective sleep parameters (LPS, WASO, TST and SE, as evaluated by Zmachine) from baseline to weeks 4 and 12 .

\section{Secondary outcomes}

The secondary outcomes are: (1) the amount of change in subjective sleep parameters (sSOL, sWASO, sTST and sSE, as derived from sleep diary data) from baseline to weeks 4 and 12, and (2) the relationship between the changes in subjective or objective sleep parameters and the ALPS-index at week 12.

\section{Exploratory outcomes}

The exploratory outcomes are: (1) relationships between the ALPS-index and changes in other scores from baseline to weeks 4 and 12, such as cognitive function (CPT, WCST, TMT and MMSE-J), questionnaires (PSQI, ESS, BDI and TCI-HA), eye movement analysis and the intestinal flora test, and (2) the relationship between changes in objective or subjective sleep parameters (LPS/sSOL, $\mathrm{WASO} / \mathrm{sWASO}$, and TST/sTST, SE/sSE) and $\mathrm{K}_{\text {trans }}$ at week 12.

\section{Sample size rationale}

Patients aged 50 years and older with insomnia disorder according to the DSM- 5 will be recruited through online advertisements. Although the pilot study does not require a sample size calculation, the sample size was set at 30 based on the results of Study 304 (NCT02783729), a clinical trial using lemborexant, ${ }^{41}$ and a previous study examining the brain clearance system. ${ }^{62}$ In Study $304,{ }^{34}$ lemborexant $(5 \mathrm{mg}$ ) improved sleep parameters from baseline as follows: $44.86 \mathrm{~min}$ (baseline) to $25.84 \mathrm{~min}$ in the latency to persistent sleep (SD 24.253), 68.36\% (baseline) to $81.29 \%$ in SE (SD 8.800), and $113.44 \mathrm{~min}$ (baseline) to $69.10 \mathrm{~min}$ in WASO (SD 34.533). To detect a significant change from baseline to 12 weeks of treatment at a two-sided $\alpha$ level of $5 \%$ with a power of $80 \%$, 28 participants for LPS, 10 for SE, and 12 for WASO were calculated to be required.

\section{Statistical analysis}

Basic statistics for each score at each evaluation point will be calculated. Objective/subjective sleep parameters at baseline will be compared with those at weeks 4 and 12 by 
using a paired t-test. The association between objective/ subjective sleep parameters at week 12 and the ALPSindex or $\mathrm{K}_{\text {trans }}$ will be analysed by statistics such as Pearson's correlation coefficient. The relation between other parameters in exploratory outcomes at weeks 4 and 12 and the ALPS-index will also be analysed by statistics such as Pearson's correlation coefficient. We will not correct for multiple comparisons because this is a pilot trial. Statistical significance will be defined by a $\mathrm{p}<0.05$.

\section{Adverse events}

Treatment-emergent adverse events will be analysed descriptively. At visits at weeks 4 and 12, adverse events (spontaneous reports of the participant's complaints), physical exam findings, and the results of blood and urine tests, and ECG will be assessed.

\section{ETHICS AND DISSEMINATION}

The study protocol was approved by the Nagoya University Certified Review Board (2021-0079). The study will be performed at Nagoya University Hospital. Informed consent will be obtained from all participants. For privacy protection, all participants will be identified using an anonymous identification code. If any necessary experimental data are provided to a joint research institution, these will be carefully protected using only the participants' identification codes and a corresponding table. The acquisition of informed consent, the inclusion/exclusion criteria, participant eligibility and the occurrence of any adverse events will be confirmed by an independent monitor from the research organisation. The monitor will confirm whether the research procedure is carried out based on the approved procedure and ensure that the data are properly stored. An auditor unrelated to this research will check whether the experimental procedures follow the protocol prepared beforehand. The monitor and auditor will survey all data derived from the research. The findings from the research will be published in peerreviewed journals and presented at local, national and international conferences.

\section{DISCUSSION}

The aim of this study is to reveal whether improved sleep quality by lemborexant improves the function of the glymphatic system as evaluated by DTI-ALPS. To the best of our knowledge, this is the first prospective trial for elucidating the relationship between sleep and the glymphatic system in humans. In an animal model, the elimination of $\mathrm{A} \beta$ was found to be faster during sleep than awake periods because the extracellular space expands under natural sleep conditions, as well as under anaesthesia. ${ }^{20}$ The number of studies on the relationship between the glymphatic system and sleep in humans has been increasing, but previous studies have been cross-sectional ${ }^{6364}$ or not performed in a clinical setting. ${ }^{21}$ Therefore, our study may identify a longitudinal relationship between the improvement of insomnia in humans by lemborexant and the function of the glymphatic system as evaluated by the ALPS index.

This study will use Zmachine, an ambulatory sleep EEG monitor, which has shown a good concordance with PSG in various sleep parameters. ${ }^{37}$ Although laboratory PSG has been shown to be the gold standard of sleep measurement, it requires an overnight stay in a sleep laboratory with multiple electrodes and other devices that can interfere with sleeping. These factors can contribute to the 'first night effect', where sleep duration and quality in the laboratory setting can substantially differ from that typically experienced at home. ${ }^{6566}$ At-home sleep monitors may help to address this problem by allowing participants to sleep in their beds while maintaining sufficient agreement with PSG. ${ }^{39}$ The effect of lemborexant on objective sleep parameters has been evaluated by PSG, ${ }^{3467}$ but there are no objective data regarding natural sleep conditions except for subjective evaluations such as sleep diaries or questionnaires. Therefore, Zmachine will make it possible to evaluate the effect of lemborexant on objective sleep parameters under near-natural sleep conditions.

Orexin is an important neuropeptide in promoting wakefulness and regulating the sleep-wake cycle, and its role in human cognition and $\mathrm{AD}$ has been also emphasised. $A \beta$ clearance from brain ISF is affected by the sleep-wake cycle, and excessive orexin signals may disrupt the sleepwake cycle and lead to the accumulation of $A \beta$. Indeed, sleep deprivation and orexin infusion in mice increased, whereas orexin receptor antagonist infusion decreased ISF A $\beta$ levels. ${ }^{68}$ If the orexin receptor antagonist lemborexant not only improve the subjective and objective sleep parameters but also affects the ALPS index, it might provide new insights regarding the pathology, treatment and prevention of neurodegenerative disorders such as $\mathrm{AD}$ in which protein aggregation occurs in the brain. ${ }^{19}$ Considering these functions of orexin in sleep and neurodegenerative disorders, we selected lemborexant rather than BzRAs as a hypnotic in this study, the purpose of which is to clarify the relationship between improvements in insomnia and the function of the glymphatic system.

If this study can clarify the relationship between sleep and the glymphatic system, the role of sleep in the brain clearance system may be revealed, leading to an important intervention in the brain clearance system for physicians and patients. This evidence might result in more attention to sleep hygiene and a more aggressive treatment approach for insomnia.

\section{Author affiliations}

${ }^{1}$ Department of Psychiatry, Nagoya University Graduate School of Medicine, Nagoya, Aichi, Japan

${ }^{2}$ Medical Headquarters, Eisai Co Ltd, Bunkyo-ku, Tokyo, Japan

${ }^{3}$ Department of Advanced Medicine, Nagoya University Hospital, Nagoya, Aichi, Japan

${ }^{4}$ Department of Innovative Biomedical Visualization, Nagoya University Graduate School of Medicine, Nagoya, Aichi, Japan

${ }^{5}$ Department of Radiology, Nagoya University Graduate School of Medicine, Nagoya, Aichi, Japan 
Acknowledgements The authors would like to thank Maika Nishida, Yurie Nishi, Shohei Nishimoto, Yuki Kogo and Takehiro Taninaga from the Medical Headquarters at Eisai, and Margaret Moline from Eisai, who made valuable contributions to the development of the study protocol.

Contributors NO developed the study concept with KI, SM, MA, AF and MT; IO and KI wrote the first draft of the manuscript; SM, AF, MT, MA, NM, TT, SN and NO made critical revisions to the manuscript; all authors read and approved the final manuscript to be submitted.

Funding This trial was supported by Eisai (Award/Grant number is not applicable). Competing interests I0, SM, MA and NM have no conflicts of interest to declare. KI has received speakers' honoraria from Eisai, Kyowa, Meiji Seika Pharma, Otsuka, Sumitomo Dainippon, Taisho, Takeda and Towa. AF and MT are employees of Eisai, Japan. TT has received research funding from Canon Medical Systems Corporation. SN has received speakers' honoraria from Eisai, Siemens Healthcare Japan, Guerbet, GE Healthcare Pharma, Nihon Medi-Physics, FUJIFILM Toyama Chemical, Bayer and Canon Medical Systems Corporation. NO has received research support or speakers' honoraria from, or has served as a consultant to, Sumitomo Dainippon, Eisai, Otsuka, KAITEKI, Mitsubishi Tanabe, Shionogi, Eli Lilly, Mochida, DAlICHI SANKYO, Nihon Medi-Physics, Takeda, Meiji Seika Pharma, EA Pharma, Pfizer, MSD, Lundbeck Japan, Taisho Pharma, Janssen, UCB and Shionogi, Nihon Medi-Physics, Tsumura, Novartis and Astellas. Eisai funded this study and participated in the planning of this study. Eisai will also review findings, commission the study report, and review the papers before they are submitted for publication.

Patient and public involvement Patients and/or the public were not involved in the design, or conduct, or reporting, or dissemination plans of this research.

Patient consent for publication Not applicable.

Provenance and peer review Not commissioned; externally peer reviewed.

Open access This is an open access article distributed in accordance with the Creative Commons Attribution Non Commercial (CC BY-NC 4.0) license, which permits others to distribute, remix, adapt, build upon this work non-commercially, and license their derivative works on different terms, provided the original work is properly cited, appropriate credit is given, any changes made indicated, and the use is non-commercial. See: http://creativecommons.org/licenses/by-nc/4.0/.

\section{ORCID iDs}

Ippei Okada http://orcid.org/0000-0003-4862-5889

Nao Matsuyama http://orcid.org/0000-0002-0096-0832

\section{REFERENCES}

1 Winkelman JW. Clinical practice. Insomnia disorder. N Engl J Med 2015;373:1437-44.

2 Roth T. Insomnia: definition, prevalence, etiology, and consequences. J Clin Sleep Med 2007;3:S7-10.

3 Liu X, Uchiyama M, Kim K, et al. Sleep loss and daytime sleepiness in the general adult population of Japan. Psychiatry Res 2000;93:1-11.

4 Baglioni C, Battagliese G, Feige B, et al. Insomnia as a predictor of depression: a meta-analytic evaluation of longitudinal epidemiological studies. J Affect Disord 2011;135:10-19.

5 Uehli K, Mehta AJ, Miedinger D, et al. Sleep problems and work injuries: a systematic review and meta-analysis. Sleep Med Rev 2014;18:61-73.

6 Martiniuk ALC, Senserrick T, Lo S, et al. Sleep-Deprived young drivers and the risk for crash: the drive prospective cohort study. JAMA Pediatr 2013;167:647-55.

7 Connor Jet al. Driver sleepiness and risk of serious injury to car occupants: population based case control study. BMJ 2002;324:1125.

8 Miyata S, Noda A, Ozaki N, et al. Insufficient sleep impairs driving performance and cognitive function. Neurosci Lett 2010;469:229-33.

9 Kato K, Miyata S, Ando M, et al. Influence of sleep duration on cortical oxygenation in elderly individuals. Psychiatry Clin Neurosci 2017;71:44-51.

10 Miyata S, Noda A, Iwamoto K, et al. Impaired cortical oxygenation is related to mood disturbance resulting from three nights of sleep restriction. Sleep Biol Rhythms 2015;13:387-94.

11 Miyata S, Noda A, Iwamoto K, et al. Poor sleep quality impairs cognitive performance in older adults. J Sleep Res 2013;22:535-41.

12 Guarnieri B, Adorni F, Musicco M, et al. Prevalence of sleep disturbances in mild cognitive impairment and dementing disorders: a multicenter Italian clinical cross-sectional study on 431 patients. Dement Geriatr Cogn Disord 2012;33:50-8.

13 Shi L, Chen S-J, Ma M-Y, et al. Sleep disturbances increase the risk of dementia: a systematic review and meta-analysis. Sleep Med Rev 2018;40:4-16.

14 Bubu OM, Brannick M, Mortimer J, et al. Sleep, cognitive impairment, and Alzheimer's disease: a systematic review and metaanalysis. Sleep 2017;40. doi:10.1093/sleep/zsw032. [Epub ahead of print: 01 Jan 2017].

15 Spira AP, Gamaldo AA, An Y, et al. Self-Reported sleep and $\beta$-amyloid deposition in community-dwelling older adults. JAMA Neurol 2013;70:1537-43.

16 Ju Y-ES, Lucey BP, Holtzman DM. Sleep and Alzheimer disease pathology--a bidirectional relationship. Nat Rev Neurol 2014;10:115-9.

17 Nedergaard M. Neuroscience. Garbage truck of the brain. Science 2013;340:1529-30.

18 Wardlaw JM, Benveniste H, Nedergaard M, et al. Perivascular spaces in the brain: anatomy, physiology and pathology. Nat Rev Neurol 2020;16:137-53.

19 Nedergaard M, Goldman SA. Glymphatic failure as a final common pathway to dementia. Science 2020;370:50-6.

20 Tarasoff-Conway JM, Carare RO, Osorio RS, et al. Clearance systems in the brain-implications for Alzheimer disease. Nat Rev Neurol 2015;11:457-70.

21 Shokri-Kojori E, Wang G-J, Wiers CE, et al. $\beta$-Amyloid accumulation in the human brain after one night of sleep deprivation. Proc Natl Acad Sci U S A 2018;115:4483-8.

22 Ju Y-ES, Ooms SJ, Sutphen C, et al. Slow wave sleep disruption increases cerebrospinal fluid amyloid- $\beta$ levels. Brain 2017;140:2104-11.

23 Xie L, Kang H, Xu Q, et al. Sleep drives metabolite clearance from the adult brain. Science 2013;342:373-7.

24 Taoka T, Masutani Y, Kawai H, et al. Evaluation of glymphatic system activity with the diffusion Mr technique: diffusion tensor image analysis along the perivascular space (DTI-ALPS) in Alzheimer's disease cases. Jpn J Radiol 2017;35:172-8.

25 Ding X-B, Wang X-X, Xia D-H, et al. Impaired meningeal lymphatic drainage in patients with idiopathic Parkinson's disease. Nat Med 2021;27:411-8

26 Taoka T, Naganawa S. Imaging for central nervous system (CNS) interstitial fluidopathy: disorders with impaired interstitial fluid dynamics. Jpn J Radiol 2021;39:1-14.

27 Soyka M. Treatment of benzodiazepine dependence. N Engl J Med Overseas Ed 2017;376:1147-57.

28 Seppala LJ, Wermelink AMAT, de Vries M, et al. Fall-Risk-Increasing drugs: a systematic review and meta-analysis: II. psychotropics. J Am Med Dir Assoc 2018;19:371.e11-371.e17.

29 Crowe SF, Stranks EK. The residual medium and long-term cognitive effects of benzodiazepine use: an updated meta-analysis. Arch Clin Neuropsychol 2018;33:901-11.

30 Penninkilampi R, Eslick GD, Systematic Review A. A systematic review and meta-analysis of the risk of dementia associated with benzodiazepine use, after controlling for Protopathic bias. CNS Drugs 2018;32:485-97.

31 Tapiainen V, Taipale H, Tanskanen A, et al. The risk of Alzheimer's disease associated with benzodiazepines and related drugs: a nested case-control study. Acta Psychiatr Scand 2018;138:91-100.

32 Richardson K, Mattishent K, Loke YK, et al. History of benzodiazepine prescriptions and risk of dementia: possible bias due to prevalent users and covariate measurement timing in a nested case-control study. Am J Epidemiol 2019;188:1228-36.

33 Scott LJ. Lemborexant: first approval. Drugs 2020;80:425-32.

34 Rosenberg R, Murphy P, Zammit G, et al. Comparison of Lemborexant with placebo and zolpidem tartrate extended release for the treatment of older adults with insomnia disorder. JAMA Netw Open 2019;2:e1918254.

35 Kishi T, Nomura I, Matsuda Y, et al. Lemborexant vs suvorexant for insomnia: a systematic review and network meta-analysis. $J$ Psychiatr Res 2020;128:68-74.

36 Chan A-W, Tetzlaff JM, Gøtzsche PC, et al. Spirit 2013 explanation and elaboration: guidance for protocols of clinical trials. BMJ 2013:346:e7586.

37 Miyata S, Iwamoto K, Banno M, et al. Performance of an ambulatory electroencephalogram sleep monitor in patients with psychiatric disorders. J Sleep Res 2021;30:e13273.

38 Kaplan RF, Wang Y, Loparo KA, et al. Performance evaluation of an automated single-channel sleep-wake detection algorithm. Nat Sci Sleep 2014;6:113-22.

39 Wang Y, Loparo KA, Kelly MR, et al. Evaluation of an automated single-channel sleep staging algorithm. Nat Sci Sleep 2015;7:101-11. 
40 Benveniste H, Lee H, Ozturk B, et al. Glymphatic cerebrospinal fluid and solute transport quantified by MRI and PET imaging. Neuroscience 2021;474:63-79.

41 Tofts PS, Brix G, Buckley DL, et al. Estimating kinetic parameters from dynamic contrast-enhanced T(1)-weighted MRI of a diffusable tracer: standardized quantities and symbols. J Magn Reson Imaging 1999;10:223-32.

42 Naganawa S, Kawai H, Taoka T, et al. Improved 3D-real inversion recovery: a robust imaging technique for endolymphatic hydrops after intravenous administration of gadolinium. MRMS 2019;18:105-8.

43 Naganawa S, Nakane T, Kawai H, et al. Gd-Based contrast enhancement of the perivascular spaces in the basal ganglia. MRMS 2017;16:61-5.

44 Buysse DJ, Reynolds CF, Monk TH, et al. The Pittsburgh sleep quality index: a new instrument for psychiatric practice and research. Psychiatry Res 1989;28:193-213.

45 Johns MW. A new method for measuring daytime sleepiness: the Epworth Sleepiness scale. Sleep 1991;14:540-5.

46 Beck ATet al. An inventory for measuring depression. Arch Gen Psychiatry 1961;4:561-71.

47 Wilson RS, Buchman AS, Arnold SE, et al. Harm avoidance and disability in old age. Exp Aging Res 2006;32:243-61.

48 Cornblatt BA, Risch NJ, Faris G, et al. The continuous performance test, identical pairs version (CPT-IP): I. New findings about sustained attention in normal families. Psychiatry Res 1988;26:223-38.

49 Heaton RK. The Wisconsin card sorting test (manual. Odessa, Fla: Psychological Assessment Resources, 1981.

50 Kashima H, Honda T, Kato M. Neuropsychological investigation on chronic schizophrenia-aspect of its frontal functions. In: Takahashi R, Flor-Henry P, Gruzelier J, eds. Cerebral dynamics, laterality and psychopathology. Amsterdam: Elsevier, 1987: 337-45.

51 Spreen OSE. A compendium of neuropsychological tests and norms. 2nd edn. New York: Oxford University Press, 1998.

52 Folstein MF, Folstein SE, McHugh PR. "Mini-mental state". A practical method for grading the cognitive state of patients for the clinician. J Psychiatr Res 1975;12:189-98.

53 Shishido E, Ogawa S, Miyata S, et al. Application of eye trackers for understanding mental disorders: cases for schizophrenia and autism spectrum disorder. Neuropsychopharmacol Rep 2019;39:72-7.

54 Carvalho N, Laurent E, Noiret N, et al. Eye movement in unipolar and bipolar depression: a systematic review of the literature. Front Psychol 2015;6:6.
55 Woods HC, Scheepers C, Ross KA, et al. What are you looking at? moving toward an attentional timeline in insomnia: a novel semantic eye tracking study. Sleep 2013;36:1491-9.

56 Morita K, Miura K, Fujimoto M, et al. Eye movement as a biomarker of schizophrenia: using an integrated eye movement score. Psychiatry Clin Neurosci 2017;71:104-14.

$57 \mathrm{Li} \mathrm{Y,} \mathrm{Hao} \mathrm{Y,} \mathrm{Fan} \mathrm{F,} \mathrm{et} \mathrm{al.} \mathrm{The} \mathrm{role} \mathrm{of} \mathrm{microbiome} \mathrm{in} \mathrm{insomnia,}$ circadian disturbance and depression. Frontiers in Psychiatry 2018;9:669.

58 Jaggar M, Rea K, Spichak S, et al. You've got male: Sex and the microbiota-gut-brain axis across the lifespan. Front Neuroendocrinol 2020;56:100815.

59 Gupta VK, Paul S, Dutta C. Geography, ethnicity or SubsistenceSpecific variations in human microbiome composition and diversity. Front Microbiol 2017;8:1162.

60 Suzuki Y, Nishijima S, Furuta Y, et al. Long-Read metagenomic exploration of extrachromosomal mobile genetic elements in the human gut. Microbiome 2019;7:119.

61 Nishiwaki H, Hamaguchi T, Ito $\mathrm{M}$, et al. Short-Chain fatty acidproducing gut microbiota is decreased in Parkinson's disease but not in Rapid-Eye-Movement sleep behavior disorder. mSystems 2020;5:e00797-20. doi:10.1128/mSystems.00797-20

62 Taoka T, Naganawa S. Glymphatic imaging using MRI. J Magn Reson Imaging 2020;51:11-24.

63 Ju Y-ES, Finn MB, Sutphen CL, et al. Obstructive sleep apnea decreases central nervous system-derived proteins in the cerebrospinal fluid. Ann Neurol 2016;80:154-9. doi:10.1002/ ana.24672

64 Varga AW, Wohlleber ME, Giménez S, et al. Reduced slow-wave sleep is associated with high cerebrospinal fluid $A \beta 42$ levels in cognitively normal elderly. Sleep 2016;39:2041-8.

65 Byun J-H, Kim KT, Moon H-jin, et al. The first night effect during polysomnography, and patients' estimates of sleep quality. Psychiatry Res 2019;274:27-9.

66 Le Bon O, Staner L, Hoffmann G, et al. The first-night effect may last more than one night. J Psychiatr Res 2001;35:165-72.

67 Murphy P, Moline M, Mayleben D, et al. Lemborexant, a dual orexin receptor antagonist (DORA) for the treatment of insomnia disorder: results from a Bayesian, adaptive, randomized, double-blind, placebo-controlled study. J Clin Sleep Med 2017;13:1289-99.

68 Kang J-E, Lim MM, Bateman RJ, et al. Amyloid-Beta dynamics are regulated by orexin and the sleep-wake cycle. Science 2009;326:1005-7. 